\title{
Nucleation in scale-free networks
}

\author{
Hanshuang Chen ${ }^{1}$, Chuansheng Shen ${ }^{1,2}$, Zhonghuai Hou ${ }^{*}$ and Houwen Xin ${ }^{1}$ \\ ${ }^{1}$ Hefei National Laboratory for Physical Sciences at Microscales and Department of Chemical Physics, \\ University of Science and Technology of China, Hefei, 230026, China \\ ${ }^{2}$ Department of Physics, Anqing Teachers College, Anqing 246011, China
}

(Dated: November 1, 2018)

\begin{abstract}
We have studied nucleation dynamics of the Ising model in scale-free networks with degree distribution $P(k) \sim k^{-\gamma}$ by using forward flux sampling method, focusing on how the network topology would influence the nucleation rate and pathway. For homogeneous nucleation, the new phase clusters grow from those nodes with smaller degree, while the cluster sizes follow a power-law distribution. Interestingly, we find that the nucleation rate $R_{H o m}$ decays exponentially with the network size $N$, and accordingly the critical nucleus size increases linearly with $N$, implying that homogeneous nucleation is not relevant in the thermodynamic limit. These observations are robust to the change of $\gamma$ and also present in random networks. In addition, we have also studied the dynamics of heterogeneous nucleation, wherein $w$ impurities are initially added, either to randomly selected nodes or to targeted ones with largest degrees. We find that targeted impurities can enhance the nucleation rate $R_{H e t}$ much more sharply than random ones. Moreover, $\ln \left(R_{H e t} / R_{H o m}\right)$ scales as $w^{\gamma-2 / \gamma-1}$ and $w$ for targeted and random impurities, respectively. A simple mean field analysis is also present to qualitatively illustrate above simulation results.

PACS numbers: 89.75.Hc, 64.60.Q-, 05.50.+q
\end{abstract}

\section{INTRODUCTION}

Complex networks describe not only the pattern discovered ubiquitously in real world, but also provide a unified theoretical framework to understand the inherent complexity in nature [1-4]. Many real networks, as diverse as ranging from social networks to biological networks to communication networks, have been found to be scale-free [5], i.e., their degree distributions follow a power-law, $P(k) \sim k^{-\gamma}$. A central topic in this field has been how the network topology would influence the dynamics taking place on it. Very recently, critical phenomena in scale-free networks (SFNs) have attracted considerable research interest [6], such as order-disorder transitions 7 -10], percolation [1 14], epidemic spreading 15], synchronization [16, 17], self-organized criticality [18, 19], nonequilibrium pattern formation [20], and so on. These studies have revealed that network heterogeneity, characterized by diverse degree distributions, makes the critical behaviors of SFNs quite different from those on regular lattices. However, most previous studies focused on evaluating the onset of phase transition in different network models. There is little attention paid to the dynamics/kinetics of phase transition, such as nucleation and phase separation in complex networks.

Nucleation is a fluctuation-driven process that initiates the decay of a metastable state into a more stable one 21]. A first-order phase transition usually involves the nucleation and growth of a new phase. Many important phenomena in nature, including crystallization 22], glass formation 23], and protein folding [24], etc., are

*Electronic address: hzhlj@ustc.edu.cn associated with nucleation. Despite its apparent importance, many aspects of nucleation process are still unclear and deserve more investigations. The Ising model, which is a paradigm for many phenomena in statistical physics, has been widely used to study the nucleation process. Despite its simplicity, the Ising model has made important contributions to the understanding of nucleation phenomena in equilibrium systems and is likely to yield important insights also for nonequilibrium systems. In two-dimensional lattices, for instance, shear can enhance the nucleation rate and at an intermediate shear rate the nucleate rate peaks [25], a single impurity may considerably enhance the nucleation rate [26], and the existence of a pore may lead to two-stage nucleation and the overall nucleation rate can reach a maximum level at an intermediate pore size. Nucleation pathway of Ising model in three-dimensional lattice has also been studied using transition path sampling approach 27]. In addition, Ising model has been frequently used to test the validity of classical nucleation theory (CNT) 28 32]. However, all these studies are limited to regular lattices in Euclidean space. Since many real systems can be modeled by complex networks, it is thus natural to ask how the topology of a networked system would influence the nucleation process of Ising model. To the best of our knowledge, this has never been studied in the literature so far.

Although the main motivation of the present study is to address a fundamental problem in statistical physics, it may also be of practical interest. For example, our study may help understand how public opinion or belief changes in the social context [33], where binary spins can represent two opposite opinions and the concept of physical temperature corresponds to a measure of noise due to imperfect information or uncertainty on the part 
of the agent. Another example is functional transition in real biological networks, such as the transition between different dynamical attractors in neural networks 34], wherein the two states of the Ising model may correspond to a neuron being fired or not. Other examples include gene regulatory networks, wherein genes can be on or off, corresponding to the two states of Ising model [35].

In the present work, we have studied nucleation process of the Ising model in SFNs. Since nucleation is an activated process that occurs extremely slow, brute-force simulation is prohibitively expensive. To overcome this difficulty, we adopt a recently developed forward flux sampling (FFS) method to obtain the rate and pathway for nucleation [36]. For homogeneous nucleation, we find that the nucleation begins with nodes with smaller degree, while nodes with larger degree are more stable. We show that the nucleation rate decays exponentially with the network size $N$, and accordingly the critical nucleus size increases linearly with $N$, implying that homogeneous nucleation can only occur in finite-size networks. Comparing the results of networks with different $\gamma$ and those of random networks, we conclude that network heterogeneity is unfavorable to nucleation. In addition, we have also investigated heterogeneous nucleation by adding impurities into the networks. It is found that the dependence of the nucleation rate on the number of random impurities is significantly different from the case of targeted impurities. These simulation results may be qualitatively understood in a mean-field manner.

The rest of the paper is organized as follows. In Sec II we give the details of our simulation model and the FFS method applied to this system. In SecIII we present the results for the nucleation rate and pathway. We then show, via both simulation and analysis, that the systemsize effect of the nucleation rate and heterogeneous nucleation. At last, discussion and main conclusions are addressed in Sec IV

\section{MODEL AND SIMULATION DETAILS}

\section{A. The networked Ising model}

The Ising model in a network comprised of $N$ nodes is described by the Hamiltonian

$$
H=-J \sum_{i<j} a_{i j} s_{i} s_{j}-h \sum_{i} s_{i}
$$

where spin variable $s_{i}$ at node $i$ takes either +1 (up) or -1 (down). $J$ is the coupling constant and $h$ is the external magnetic field. The element of the adjacency matrix of the network takes $a_{i j}=1$ if nodes $i$ and $j$ are connected and 0 otherwise.

Our simulation is performed by Metropolis spin-flip dynamics [37], in which we attempt to flip each spin once, on average, during each Monte Carlo (MC) cycle. In each attempt, a randomly chosen spin is flipped with the probability $\min \left(1, e^{-\beta \Delta E}\right)$, where $\beta=1 /\left(k_{B} T\right)$ with $k_{B}$ the Boltzmann constant and $T$ the temperature, $\Delta E$ is the energy change due to the flipping process. Generally, with the increment of $T$, the system will undergo a second-order phase transition at the critical temperature $T_{c}$ from an ordered state to a disordered one. To study nucleation, we set $J=1, h>0, T<T_{c}$, and start from a metastable state in which $s_{i}=-1$ for most of the spins. The system will stay in that state for a significantly long time before undergoing a nucleation transition to the thermodynamic stable state with most spins pointing up. We are interested in dynamics of this nucleation process.

\section{B. Forward flux sampling}

FFS method has been used to calculate rate constants, transition paths and stationary probability distributions for rare events in equilibrium and nonequilibrium systems [25, 26, 36, 38 40]. This method uses a series of interfaces in phase space between the initial and final states to force the system from the initial state $A$ to the final state $B$ in a ratchet-like manner. An order parameter $\lambda(x)$ is first defined, where $x$ represents the phase space coordinates, such that the system is in state $A$ if $\lambda(x)<\lambda_{0}$ and state $B$ if $\lambda(x)>\lambda_{m}$, while a series of nonintersecting interfaces $\lambda_{i}(0<i<m)$ lie between states $A$ and $B$, such that any path from $A$ to $B$ must cross each interface without reaching $\lambda_{i+1}$ before $\lambda_{i}$. The transition rate $R$ from $A$ to $B$ is calculated as

$$
R=\bar{\Phi}_{A, 0} P\left(\lambda_{m} \mid \lambda_{0}\right)=\bar{\Phi}_{A, 0} \prod_{i=0}^{m-1} P\left(\lambda_{i+1} \mid \lambda_{i}\right),
$$

where $\bar{\Phi}_{A, 0}$ is the average flux of trajectories crossing $\lambda_{0}$ in the direction of $B . P\left(\lambda_{m} \mid \lambda_{0}\right)=\prod_{i=0}^{m-1} P\left(\lambda_{i+1} \mid \lambda_{i}\right)$ is the probability that a trajectory crossing $\lambda_{0}$ in the direction of $B$ will eventually reach $B$ before returning to $A$, and $P\left(\lambda_{i+1} \mid \lambda_{i}\right)$ is the probability that a trajectory which reaches $\lambda_{i}$, having come from $A$, will reach $\lambda_{i+1}$ before returning to $A$. For more information about FFS, please turn to Ref.[41].

\section{RESULTS}

\section{A. Homogeneous Nucleation: Rate and pathway}

To begin, we first consider homogeneous nucleation in Barabási-Albert scale-free network (BA-SFN) whose degree distribution follows a power-law $P(k) \sim k^{-\gamma}$ with the scaling exponent $\gamma=3[5]$. We define the order parameter $\lambda$ as the total number of up spins in the networks. We set $N=1000$, the average degree $\langle k\rangle=6$, $T=2.59, h=0.7, \lambda_{0}=130$ and $\lambda_{m}=880$, where $T$ is lower than the critical temperature $T_{c} \simeq 10.36$. The spacing between interfaces is fixed at 3 up spins, but the computed results do not depend on this spacing. During 


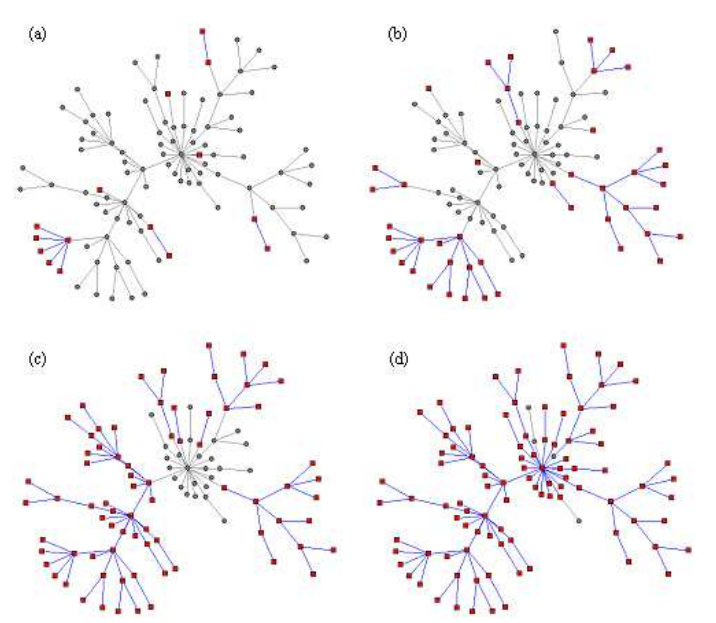

FIG. 1: (Color online) Snapshots of nucleation in a BA scalefree network with $N=100$ and $\langle k\rangle=2$ at four different stages. Up-spins and down-spins are indicated by red squares and black circles, respectively.

the FFS sampling, we perform 1000 trials at each interface, from which at least 100 configurations are stored in order to investigate the statistical properties of the ensemble of nucleation pathway. We obtain $\bar{\Phi}_{A, 0}=1.24 \times$ $10^{-4} M$ Cstep $^{-1}$ spin $^{-1}$ and $P\left(\lambda_{m} \mid \lambda_{0}\right)=4.48 \times 10^{-46}$, resulting in $R_{\text {Hom }}=5.55 \times 10^{-50} \mathrm{MCstep}^{-1}$ spin $^{-1}$ following Eq.2. Such a nucleation rate is very low such that a brute-force simulation would be very expensive.

From the stored configurations at each interface, one can figure out the details of the nucleation pathway. Figure 1 illustrates schematically four stages of a typical nucleation pathway. Clearly, the new phase (indicated by squares) starts from nodes with smaller degree, while nodes with larger degree are more stable. This picture is reasonable because nodes with larger degrees need to overcome more interfacial energies. Figure 2(a) plots the average degree of network nodes in the new phase, $\left\langle k_{n e w}\right\rangle$, as a function of the order parameter $\lambda$. As expected, $\left\langle k_{n e w}\right\rangle$ increases monotonously with $\lambda$. On the other hand, it is observed that the formation of large clusters of new phase is accompanied with the growth and coalescence of small clusters. Interestingly, we find that the size $N_{c}$ of new phase clusters follows a power law distribution at early stages of nucleation, $P\left(N_{c}\right) \sim N_{c}^{-\alpha}$ with the fitting exponent $\alpha \simeq 2.44$, as shown in Fig 2 (b). With the emergence of a giant component of new phase, the tail of the distribution is elevated, but the size distribution for the remaining clusters still follows power-law. The underlying mechanism of such phenomenon is still an open question for us.

To determine the critical size of the nucleus, $\lambda_{c}$, we compute the committor probability $P_{B}$, which is the probability of reaching the thermodynamic stable state before returning to the metastable state. The dependence of $P_{B}$ on $\lambda$ is plotted in Fig 3(a). As commonly
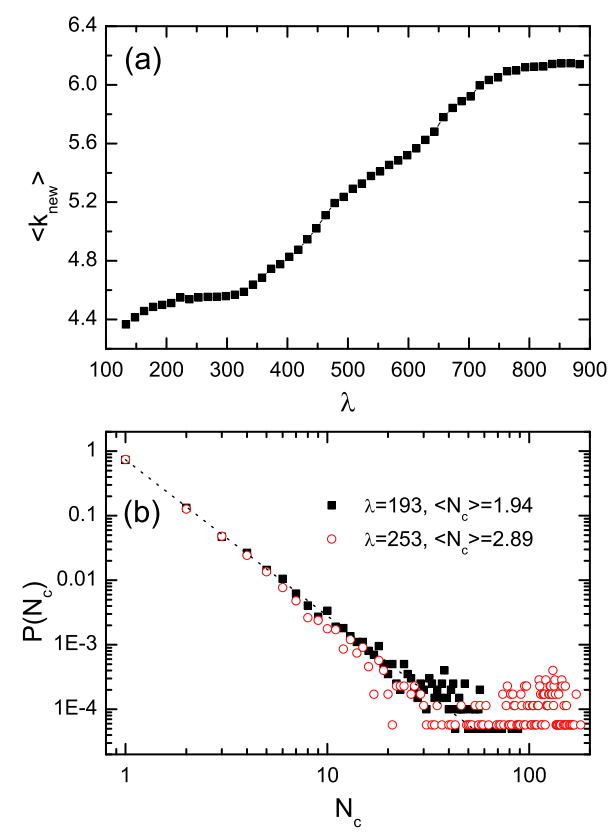

FIG. 2: (Color online) (a) The average degree of nodes of new phase $\left\langle k_{n e w}\right\rangle$ as a function of $\lambda$; (b) The size distribution of clusters of new phase, in which a power law distribution is present. Other parameters are $N=1000,\langle k\rangle=6, T=2.59$, and $h=0.7$.

reported in the literature 27, 32], the critical nucleus appears at $P_{B}\left(\lambda_{c}\right)=0.5$, giving the critical nucleus size $\lambda_{c}^{F F S}=474$. The committor distribution at $\lambda_{c}^{F F S}$ exhibits a peak at 0.5 , of which $70 \%$ of spin configurations have $P_{B}$ values within the range of 0.4 to 0.6 (see the inset of Fig $3(\mathrm{a})$ ), indicating that $\lambda$ is a proper order parameter.

Note that conventionally the nucleation threshold $\lambda_{c}$ is usually estimated by using CNT [42 44]. One can calculate the free energy change along the nucleation path, $\Delta F(\lambda)$, by using methods like umbrella sampling (US) [45]. According to CNT, $\Delta F$ will bypass a maximum at $\lambda=\lambda_{c}^{U S}$, and the nucleation rate is given by $\nu \exp \left(-\beta \Delta F_{c}\right)$, where $\nu$ is an attempt frequency. Here we have computed $\Delta F$ by using US, in which we have adopted a bias potential $0.1 k_{B} T(\lambda-\bar{\lambda})^{2}$, with $\bar{\lambda}$ being the center of each window. As shown in Fig 3 (b), the maximum in $\Delta F$ occurs at $\lambda_{c}^{U S}=451$, giving a freeenergy barrier of $\Delta F_{c} \simeq 91.4 k_{B} T$. Clearly $\lambda_{c}^{U S}$ gives a fairly good estimation of $\lambda_{c}^{F F S}$. To calculate the nucleation rate, however, one has to obtain the attempt frequency $\nu$, which is not a trivial task. If we just set $\nu=1$, we obtain a CNT prediction of a rate of $2.02 \times 10^{-40}$ MCstep $^{-1}$ spin $^{-1}$, which is 9 orders of magnitude faster than that computed from FFS method. This level of disagreement in nucleation rate corresponds to an error in the free-energy barrier of about $24 \%$. Since the accurate value of $\nu$ is generally unavailable, we will use FFS method to calculate the nucleation rate throughout this paper. In addition, real nucleation pathway cannot 

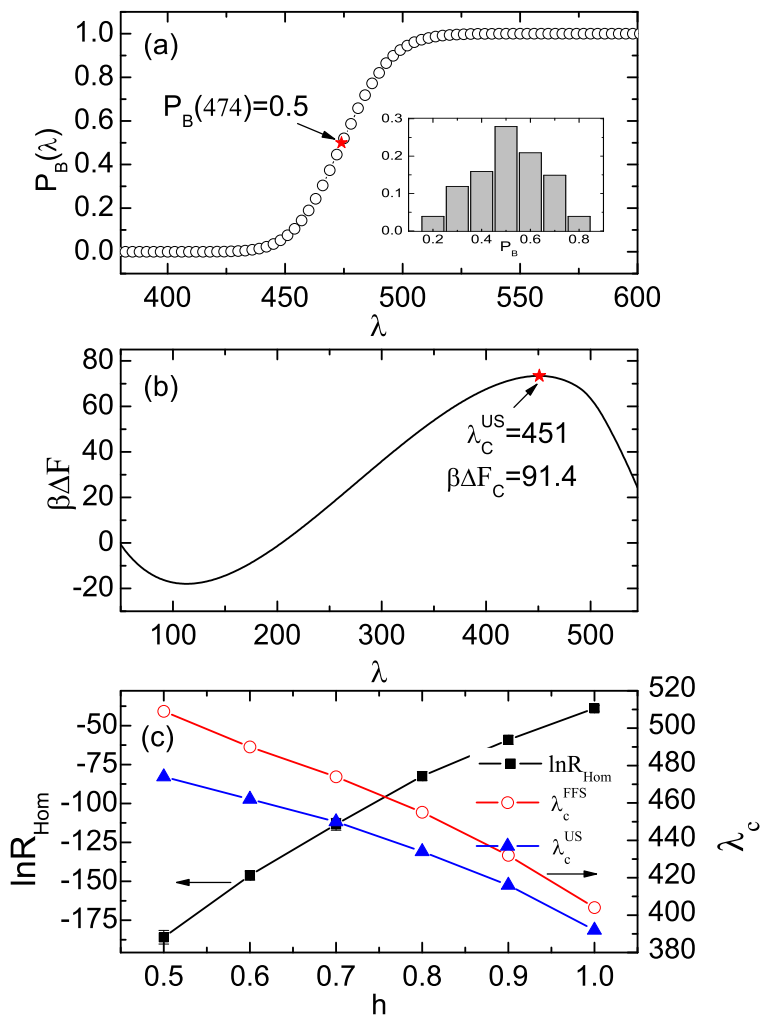

FIG. 3: (Color online) (a) The committor probability $P_{B}$ as a function of $\lambda$; The inset plots the committor distribution at $\lambda_{c}^{F F S}$. (b) The free energy $\Delta F$ as a function of $\lambda$, in which the maximum in $\Delta F$ occurs at $\lambda_{c}^{U S}$. (c) The logarithm of homogeneous nucleation rate $\ln R_{H o m}$ (left axis), and the critical size of nucleus, $\lambda_{c}^{F F S}$ and $\lambda_{c}^{U S}$ (right axis), obtained by FFS method and US method, respectively, as functions of $h$. Other parameters are the same as Fig 2.

be obtained by conventional US method due to the use of a biased potential.

We have also investigated how the nucleation rate and threshold depend on the external field $h$. In Fig 3 (c), $\ln R_{H o m}, \lambda_{c}^{F F S}$ and $\lambda_{c}^{U S}$ are plotted as functions of $h$. The error-bars are obtained via 20 different network realizations and 10 independent FFS samplings. As expected, $\ln R_{H o m}$ increases monotonously with $h$, and $\lambda_{c}^{F F S}$ and $\lambda_{c}^{U S}$ both decrease with $h$. For large $h$, the difference between $\lambda_{c}^{F F S}$ and $\lambda_{c}^{U S}$ becomes small. If $h$ is large enough, one expects that the free-energy barrier will disappear, and nucleation will be not relevant.

\section{B. Homogeneous Nucleation: System-size effects}

According to Fig 3, one finds that nearly half of the nodes must be inverted to achieve nucleation. This means that for a large network, nucleation is very difficult to occur. An interesting question thus arises: How the nucleation rate and threshold depend on the network size?

To answer this question, we have performed extensive simulations to calculate $R_{H o m}$ and $\lambda_{c}$ for different network size $N$. In particular, besides the BA-SFNs, we have also considered different network types, including SFNs with other scaling exponent $\gamma$ and homogeneous random networks (HoRNs) [46]. The networks are generated according to the Molloy-Reed model [47]: Each node is assigned a random number of stubs $k$ that are drawn from a specified degree distribution. This construction eliminates the degree correlations between neighboring nodes. We note here that the exponent $\gamma$ can be a measure of degree heterogeneity of the network, i.e., the smaller $\gamma$ is, the degree distribution is more heterogeneous. In a HoRN, each node is equivalently connected to other $\langle k\rangle$ nodes, randomly selected from the whole network, such that no degree heterogeneity exists. By comparing the results in SFNs with different $\gamma$ as well as that in HoRNs, one can on one hand, check the robustness of the system size effects, and on the other hand, investigate how the degree heterogeneity affects the nucleation process.

Figure 4 shows the simulation results. All the parameters are the same as in Fig.2, expect that $N$ varies from $N=500$ to $N=3000$. Interestingly, both $\ln R_{\text {Hom }}$ and $\lambda_{c}$ show very good linear dependences on the system size, i.e., $\ln R_{H o m} \sim-a N$ and $\lambda_{c} \sim b N$ with $a$ and $b$ being positive constants. Obviously, in the thermodynamic limit $N \rightarrow \infty$, we have $R_{H o m} \rightarrow 0$ and $\lambda_{c} \rightarrow \infty$. This means that nucleation in these systems is not relevant in the thermodynamic limit, and only finite-size systems are of interest. As shown in Fig.4, for the network types considered here, qualitative behaviors are the same. Quantitatively, with increasing $\gamma$, the line slope becomes smaller, $R_{\text {Hom }}$ becomes larger and $\lambda_{c}$ gets smaller. Since larger $\gamma$ corresponds to more homogeneous degree distribution, these results indicate that the degree heterogeneity is unfavorable to nucleation. This is consistent with the nucleation pathway as shown in Fig.2: In a heterogenous network, those hub nodes are difficult to flip, making the nucleation difficult.

In the following, we will show that the system-size effects can be qualitatively understood by CNT and simple mean-field (MF) analysis. According to CNT, the formation of a nucleus lies in two competing factors: the energy cost of creating a new up spin which favors the growth of the nucleus, and an opposing factor which is due to the creation of new interfaces between up and down spins. The change in the free energy may be written as [42 44]

$$
\Delta F(\lambda)=-2 h \lambda+\sigma \lambda,
$$

where $\sigma$ denotes the effective interfacial free energy, which may depend on $T, h$, and $N$. Since interfacial interactions arise from up spins inside the nucleus and down spins outside it, one may write $\sigma=2 J K_{\text {out }}$ by neglecting entropy effects (zero-temperature approximation), where $K_{\text {out }}$ is the average number of neighboring down-spin nodes that an up-spin node has. Using MF approximation, one has $K_{\text {out }}=\langle k\rangle(1-\lambda / N)$. Inserting this relation to $\mathrm{Eq} 3$ and maximizing $\Delta F$ with respect to 


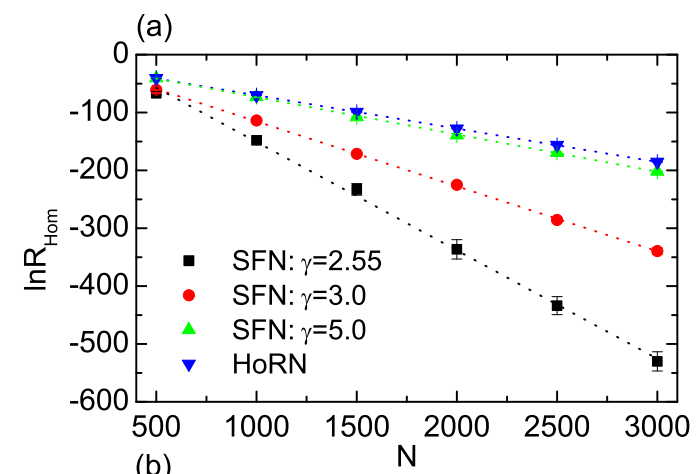

(b)

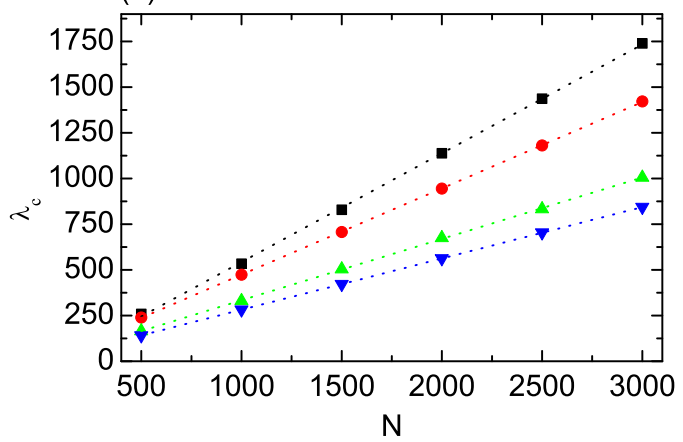

FIG. 4: (Color online) (a) The logarithm of homogeneous nucleation rate $\ln R_{H o m}$ and (b) the critical size of nucleus $\lambda_{c}$ as functions of the network size $N$ in SFNs with different $\gamma$ and in HoRNs. Other parameters are the same as Fig.2.

$\lambda$, we have

$$
\lambda_{c}^{M F}=\frac{J\langle k\rangle-h}{2 J\langle k\rangle} N,
$$

and the free-energy barrier

$$
\Delta F_{c}^{M F}=\frac{(J\langle k\rangle-h)^{2} N}{2 J\langle k\rangle} .
$$

Clearly, both $\lambda_{c}^{M F}$ and $\Delta F_{c}^{M F}$ linearly increase with $N$ if other parameters are fixed. Therefore, the linear relationships shown in Fig 4 are essentially analogous to the behavior of a mean-field network. Quantitatively, however, the MF analysis fails to predict the line slopes in Fig 4 This can be understood because the approximations are so crude, wherein important aspects such as network heterogeneity and entropy effects have not been accounted for. A rigid analysis is not a trivial task and beyond the scope of the present work.

\section{Heterogeneous nucleation}

In practice, most nucleation events that occur in nature are heterogeneous, i.e., impurities of the new phase are initially present. It is well known that impurities can increase nucleation rate by as much as several orders of magnitude. In our model, impurities are introduced by fixing some nodes in up-spin state. We are interested in how the number $w$ of impurity nodes and the way of adding impurities would affect the nucleation rate. The first way of adding impurities we use is that impurity nodes are selected in a random fashion. Figure 5 (a) gives the simulation results of $\ln \left(\frac{R_{H e t}}{R_{H o m}}\right)$ as a function of $w$ in different network models, where $R_{H e t}$ are the rates of heterogeneous nucleation. As expected, nucleation becomes faster in the presence of random impurities no matter which kind of network model is applied. It seems that in Fig 5 (a) all data collapse and exhibit a linear dependence on $w$, with the fitting slope 3.33. This means that each additional random impurity can lead to the increase of the rate by more than one order of magnitude. For the second way, we select $w$ nodes with most highly degree as the impurity nodes, termed as targeted impurities. Strikingly, such a targeted scheme is much more effective in increasing the nucleation rate than random one, as shown in Fig 5(b). For example, for SFNs with $\gamma=3$, one single targeted impurity can increase the rate by about 36 orders of magnitude.

As in Sec IIIB, below we will also give a MF analysis of the heterogeneous nucleation, which qualitatively agrees with the simulation results. Each impurity node contributes an additional term to the free energy barrier, which can, under zero-temperature approximation, be written as the product of $-2 J$ and the expected degree of the impurity node. For random impurities, each impurity node has an expected degree $\langle k\rangle$, yielding the term $-2 J\langle k\rangle$. Thus, the resulting free-energy barrier of the heterogenous nucleation becomes $\Delta_{1} F_{c}^{H e t}=$ $\Delta F_{c}^{H o m}-2 J\langle k\rangle w$, where $\Delta F_{c}^{H o m}$ is the free-energy barrier of homogeneous nucleation. According to CNT, one obtains

$$
\ln \left(\frac{R_{H e t}}{R_{H o m}}\right)=\frac{2 J\langle k\rangle}{k_{B} T} w .
$$

Therefore, nucleation with random impurities is always faster than without impurity, and $\ln \left(\frac{R_{H e t}}{R_{H o m}}\right)$ should vary linearly with $w$. The theoretical estimate of the slope is given by $2 J\langle k\rangle / k_{B} T=4.63$, approximately consistent with the simulation one. Given the simple nature of the above approximation the agreement is satisfactory. For the targeted way, a similar treatment to the former case can also be executed, except that $\langle k\rangle$ should be replaced by $\langle k\rangle_{w}$, where $\langle k\rangle_{w}$ is the average degree of the $w$ targeted nodes. After simple calculations, we can obtain $\langle k\rangle_{w}=\langle k\rangle\left(\frac{N}{w}\right)^{\frac{1}{\gamma-1}}$. This leads to a free-energy barrier, $\Delta_{2} F_{c}^{H e t}=\Delta_{2} F_{c}^{H o m}-2 J\langle k\rangle N^{\frac{1}{\gamma-1}} w^{\frac{\gamma-2}{\gamma-1}}$, and

$$
\ln \left(\frac{R_{H e t}}{R_{\text {Hom }}}\right)=\frac{2 J\langle k\rangle}{k_{B} T} N^{\frac{1}{\gamma-1}} w^{\frac{\gamma-2}{\gamma-1}} .
$$

Compared with Eq 6 besides the presence of an additional size-dependent factor of $N^{\frac{1}{\gamma-1}}$, the $w$-dependent factor becomes $w^{\frac{\gamma-2}{\gamma-1}}$ rather than $w$. For homogeneous 
(a) random way

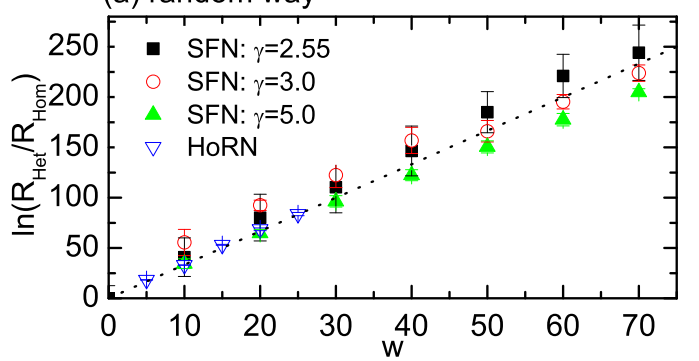

(b) targeted way

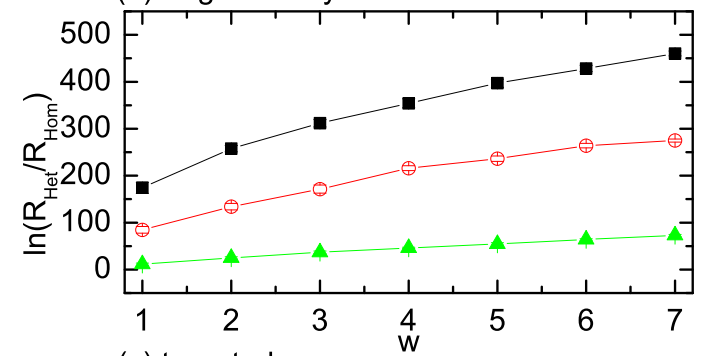

(c) targeted way

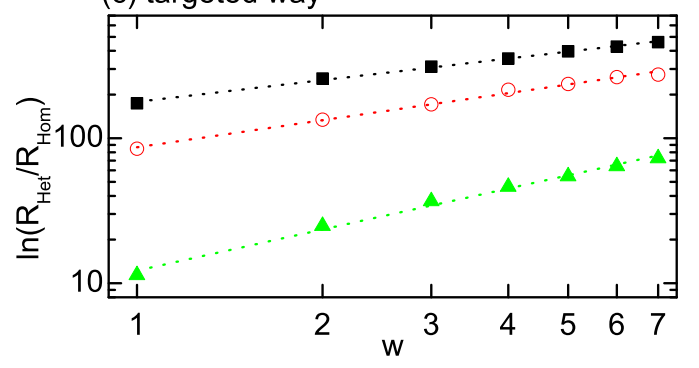

FIG. 5: (Color online) $\ln \left(\frac{R_{\text {Het }}}{R_{\text {Hom }}}\right)$ as a function of the number of impurity nodes $w$. (a) corresponds to the case of random impurities, while (b) and (c) to the case of targeted impurities. (c) plots $\ln \left(\frac{R_{H e t}}{R_{\text {Hom }}}\right)$ vs $w$ in double logarithmic coordinates. All dotted lines are drawn by linear fitting. Other parameters are the same as Fig 2 except for $h=0.2$.

network, i.e. $\quad \gamma \rightarrow \infty$, one obtains $N^{\frac{1}{\gamma-1}} \rightarrow 1$ and $w^{\frac{\gamma-2}{\gamma-1}} \rightarrow w$, and Eq[7 is thus equivalent to Eq[6. In Fig 5 (c), we plot the simulation results of $\ln \left(\frac{R_{\text {Het }}}{R_{\text {Hom }}}\right)$ as a function of $w$ in double logarithmic coordinates, where linear dependences are apparent, in agreement with the result of Eq,7. The fitting slopes $\mu$ and intercepts $\kappa$ for $\gamma=2.55,3.0,5.0$ in Fig 5(c) are $\mu=0.49,0.62,0.93$, and $\kappa=2.25,1.94,1.08$, respectively, while our analytical estimates are $\mu=\frac{\gamma-2}{\gamma-1}=0.35,0.50,0.75$, and $\kappa=\log \left(\frac{2 J\langle k\rangle N^{\frac{1}{\gamma-1}}}{k_{B} T}\right)=2.60,2.16,1.42$, respectively. Simulations and analysis give the same trends of the values of $\mu, \kappa$ and $\gamma$.

\section{DISCUSSION AND CONCLUSIONS}

Our investigations of system-size effects of nucleation in SFNs and HoRNs have revealed that the nucleation rate is size-dependent, and it decreases exponentially with the network size, resulting in that nucleation only occurs at finite-size systems. As we already know, such system-size dependence does not exist in two-dimensional regular lattices [26]. We have also studied nucleation of Ising system in regular networks where each node is connected to its $k$-nearest neighbors (here we only consider the case of sparse networks, i.e., $k \ll N$ ), and found that the rate is almost independent of network size (results not shown). Therefore, nucleation process in SFNs and HoRNs are quite different from that in regular lattices or networks. Such differences may originate from the infinite-dimensional properties of SFNs and HoRNs, wherein the average path distance is rather small, rendering the system's behavior analogous to that of a meanfield network. An interesting situation arises when one considers Watts-Strogatz small-world network, which is constructed by randomly rewiring each link of a regular network with the probability $p$ [48]. With the increment of $p$ from 0 to 1 , the resulting network changes from a regular network to a completely random one. As mentioned above, for the nucleation process, no system-size effects exist for $p=0$, while system-size dependence exists for $p=1$. One may naturally ask: How does the crossover happens when $p$ changes from 0 to 1 , and what is the physical significance of such a transition? This question surely deserves further investigations and may be the content of a future publication.

In summary, we have studied homogeneous and heterogeneous nucleation of Ising model in SFNs, by using FFS method. For homogeneous nucleation, we find that the formation of new phase starts from nodes with smaller degree, while nodes with higher degree are more stable. Extensive simulations show that the nucleation rate decreases exponentially with the network size $N$, and the nucleation threshold increases linearly with $N$, indicating that nucleation in these systems are not relevant in the thermodynamic limit. For heterogeneous nucleation, target impurities are shown to be much more efficient to enhance the nucleation rate than random ones. Simple $\mathrm{MF}$ analysis is also present to qualitatively illustrate the simulation results. Our study may provide valuable understanding how first-order phase transition takes place in network-organized systems, and how to effectively control the rate of such a process.

\section{Acknowledgments}

This work is supported by the National Science Foundation of China under Grants No. 20933006 and No. 20873130 . 
[1] R. Albert and A.-L. Barabási, Rev. Mod. Phys. 74, 47 (2002).

[2] S. Boccaletti, V. Latora, Y. Moreno, M. Chavez, and D.U. Hwang, Phys. Rep. 424, 175 (2006).

[3] A. Arenas, A. Díaz-Guilera, J. Kurths, Y. Moreno, and C. Zhou, Phys. Rep. 469, 93 (2008).

[4] M. E. J. Newman, SIAM Review 45, 167 (2003).

[5] A.-L. Barabási and R. Albert, Science 286, 509 (1999).

[6] S. N. Dorogovtsev, A. V. Goltseve, and J. F. F. Mendes, Rev. Mod. Phys. 80, 1275 (2008).

[7] A. Aleksiejuk, J. A. Holysta, and D. Stauffer, Physica A 310, 260 (2002).

[8] G. Bianconi, Phys. Lett. A 303, 166 (2002).

[9] S. N. Dorogovtsev, A. V. Goltsev, and J. F. F. Mendes, Phys. Rev. E 66, 016104 (2002).

[10] M. Leone, A.Vázquez, A. Vespignani, and R. Zecchina, Eur. Phys. J. B 28, 191 (2002).

[11] R. Cohen, K. Erez, D. ben Avraham, and S. Havlin, Phys. Rev. Lett. 85, 4626 (2000).

[12] D. S. Callaway, M. E. J. Newman, S. H. Strogatz, and D. J. Watts, Phys. Rev. Lett. 85, 5468 (2000).

[13] M. E. J. Newman, Phys. Rev. Lett. 89, 208701 (2002).

[14] R. Cohen, D. ben Avraham, and S. Havlin, Phys. Rev. E 66, 036113 (2002).

[15] R. Pastor-Satorras and A. Vespignani, Phys. Rev. Lett. 86, 3200 (2001).

[16] T. Nishikawa, A. E. Motter, Y.-C. Lai, and F. C. Hoppensteadt, Phys. Rev. Lett. 91, 014101 (2003).

[17] J. Gómez-Gardeñes, Y. Moreno, and A. Arenas, Phys. Rev. Lett. 98, 034101 (2007).

[18] K.-I. Goh, D.-S. Lee, B. Kahng, and D. Kim, Phys. Rev. Lett. 91, 148701 (2003).

[19] A. E. Motter and Y. C. Lai, Phys. Rev. E 66, 065102 (2002).

[20] H. Nakao and A. S. Mikhailov, Nat. Phys. 6, 544 (2010).

[21] D. Kashchiev, Nucleation: basic theory with applications (Butterworths-Heinemann, Oxford, 2000).

[22] L. Gránásy and F. Iglói, J. Chem. Phys. 107, 3634 (1997).

[23] G. Johnson, A. I. Melćuk, H. Gould, W. Klein, and R. D. Mountain, Phy. Rev. E 57, 5707 (1998).

[24] A. R. Fersht, Proc. Natl. Acad. Sci. USA 92, 10869 (1995).

[25] R. J. Allen, C. Valeriani, S. Tanase-Nicola, P. R. ten Wolde, and D. Frenke, J. Chem. Phys. 129, 134704
(2008).

[26] R. P. Sear, J. Phys. Chem. B 110, 4985 (2006).

[27] A. C. Pan and D. Chandler, J. Phys. Chem. B 108, 19681 (2004).

[28] M. Acharyya and D. Stauffer, Eur. Phys. J. B 5, 571 (1998).

[29] V. A. Shneidman, K. A. Jackson, and K. M. Beatty, J. Chem. Phys. 111, 6932 (1999).

[30] S. Wonczak, R. Strey, and D. Stauffer, J. Chem. Phys. 113, 1976 (2000).

[31] K. Brendel, G. T. Barkema, and H. van Beijeren, Phys. Rev. E 71, 031601 (2005).

[32] S. Ryu and W. Cai, Phys. Rev. E 81, 030601(R) (2010).

[33] C. Castellano, S. Fortunato, and V. Loreto, Rev. Mod. Phys. 81, 591 (2009).

[34] Y. Bar-Yam and I. R. Epstein, Proc. Natl. Acad. Sci. USA 101, 4341 (2004).

[35] T. Tian and K. Burrage, Proc. Natl. Acad. Sci. USA 103, 8372 (2006).

[36] R. J. Allen, P. B. Warren, and P. R. ten Wolde, Phy. Rev. Lett. 94, 018104 (2005).

[37] D. P. Landau and K. Binder, A Guide to Monte Carlo Simulations in Statistcal Physics (Cambridge University Press, Cambridge, 2000).

[38] A. J. Page and R. P. Sear, Phys. Rev. Lett. 97, 065701 (2006).

[39] C. Valeriani, R. J. Allen, M. J. Morelli, D. Frenkel, and P. R. ten Wolde, J. Chem. Phys. 127, 114109 (2007).

[40] R. J. Allen, D. Frenkel, and P. R. ten Wolde, J. Chem. Phys. 124, 024102 (2006).

[41] R. J. Allen, C. Valeriani, and P. R. ten Wolde, J. Phys.: Condens. Matter 21, 463102 (2009).

[42] R. Becker and W. Döring, Ann. Phys. (N.Y.) 24, 719 (1935).

[43] Y. B. Zeldovich, Ann. Phys. (N.Y.) 18, 1 (1943).

[44] A. Laaksonen, V. Talanquer, and D. W. Oxtoby, Annu. Rev. Phys. Chem. 46, 489 (1995).

[45] J. S. van Duijneveldt and D. Frenkel, J. Chem. Phys. 96, 15 (1992).

[46] F. C. Santos, J. F. Rodrigues, and J. M. Pacheco, Phys. Rev. E 72, 056128 (2005).

[47] M. Molloy and B. Reed, Random Struct. Algorithms 6, 161 (1995).

[48] D. J. Watts and S. H. Strogatz, Nature 393, 440 (1998). 